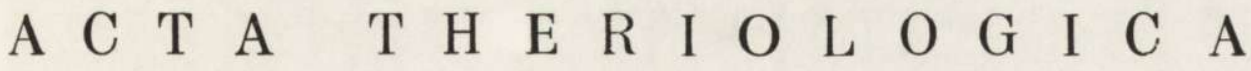 \\ VOL. XII, 9: 105-110. \\ BIAEOWIEŻA \\ 30.IV.1967
}

Piatr WYROST \& Jerzy K UCHARCZYK

\section{Versuch der Bestimmung der Widerristhöhe des Hundes mittels der inneren Hirnhöhlenlänge}

[Mit 1 Tabelle \& 1 Abb.]

\begin{abstract}
Es wird versucht die Widerristhöhe des Hundes nach den Massen der inneren Hirnhöhlenlänge zu bestimmen. Anhand von Messungen an 35 erwachsenen Hunden 19 verschiedener Rassen wurde eine Korrelation von $95,1 \%$ zwischen diesen Merkmalen festgestellt. Die praktisch besten Ergebnisse wurden mit der Regressionsgeraden $\mathrm{W}=1,016 \cdot \mathrm{D}-$ $-31,2$ erzielt, wobei $\mathrm{W}$ die Widerristhöhe in $\mathrm{cm}$ und $\mathrm{D}$ die innere Exirnhöhlenlänge in mm bedeuten. Nach dieser Formel kann die Widerristhöhe mit einem Standardfehler von $4,6 \mathrm{~cm}$ bestimmt werden.
\end{abstract}

Bei archäologischen Ausgrabungen findet man nur sehr selten gänzlich erhaltene Skelette, nach denen man die Höhe der entsprechenden Tiere bestimmen könnte. Schon seit langher werden Versuche unternommen siehe Koudelka (1886), Ki es ew a l ter (1888), Witt (1952), M ü ller (1955), Boessneck (1956) und Calkin (1961) - die Tiereshöhe auf Grund bestimmter Knochenmasse, besonders die der langen Knochen (Ossa longa) oder des Schädels, zu errechnen. Die Widerristhöhen-Quotienten werden auf zweierlei Weise bestimmt: entweder rein empirisch als Verhältnis der Tiereshöhe zur Länge der Knochenelemente (Koudelka, 1886; B oessneck, 1956), oder als Verhältnis der Mittelwerte der in Frage kommenden Merkmale (C a lk in, 1961). Wie wir feststellen werden, sind beide Methoden nicht die besten.

Das behandelte Problem ist ein typisches Beispiel der in der Statistik bekannten und fast klassichen Regressionsanalyse (P r k a l, 1963; E l a ndt, 1964). Bei Gelegenheit von anderen Untersuchungen (W yrost \& K u charczyk, 1964), welche die Analysis einiger Parameter des Hundeschädels bezüglich ihres taxonomischen Wertes betrafen, hatten wir eine grosse Korrelation zwischen Widerristhöhe des Hundes und 
der inneren Hirnhöhlenlänge (Basion - Ethmoideum) festgestellt ${ }^{1}$ ). Wir werden nun versuchen mit statistischen Regressionsmethoden das in der Praxis für die Morphologie wichtige Problem der Bestimmung der Widerristhöhe des Hundes auf Grund der inneren Hirnhöhlenlänge anzugreifen.

Als Ausgangsmaterial dienen die Messungen der inneren Hirnhöhlenlänge (D) und der Widerristhöhe (W) von 35 erwachsenen Hunden 19 verschiedener Rassen (9 Hündinnen und 26 Rüden). Die Werte der Messungen sind der Tabelle 1 zu entnehmen. Eine allgemeine Charakteristik der Messungen sieht wie folgt aus: (1) Innere Hirnhöhlenlänge $(B-E$ thm.) Bereich von 52 bis $115 \mathrm{~mm}$, Spannweite $63 \mathrm{~mm}$, Mittelwert $88 \mathrm{~mm}$, Standardfehler ${ }^{ \pm} 14 \mathrm{~mm}$, Variationskoeffizient $15,9 \%$. (2) Widerristhöhe: Bereich von 20 bis $80 \mathrm{~cm}$, Spannweite $60 \mathrm{~cm}$, Mittelwert $58,2 \mathrm{~cm}$, Standardfehler $\pm 15 \mathrm{~cm}$, Variationskoeffizient $25,7 \%$. Der Korrelationskoeffizient für diese zwei Merkmale ist sehr hoch und beträgt $95,1 \%$.

Zuerst werden wir versuchen den Quotienten für die Höhenbestimmung zu berechnen. Nach der Methode von C a lk in (1961) würden wir ihn als Verhältnis der beiden Mittelwerte erhalten, d.i. $58,2 / 88,0=0,66$. Nun aber werden wir ihn so bestimmen, wie man es in statistischen Regressionsmodellen macht.

Wir haben für jeden der 35 Hunde ein Paar der Messungen $W_{i}$ und $D_{i}$ (wobei $\mathrm{i}=1,2, \ldots, 35$ alle Hundenummern durchläuft). Wir wollen so einen Quotienten X finden, dass er, mit der inneren Hirnhöhlenlänge D multipliziert, die Widerristhöhe des Hundes ergibt nach der Gleichung $\mathrm{W}=\mathrm{X} \cdot \mathrm{D}$. Für jedes Tier einzeln ist $\mathrm{X}_{\mathrm{i}}=\mathrm{W}_{\mathrm{i}} / \mathrm{D}_{\mathrm{i}}$, aber dieses Verhältnis ändert sich von Tier zu Tier wie aus der letzten Spalte der Tabelle 1 ersichtlich ist. Wir suchen jedoch einen gemeinsamen Quotienten X für alle Hunde, demnach wird sich die nach ihm berechnete "theoretische” Widerristhöhe $\mathrm{V}_{\mathrm{i}}$ von der reellen Widerristhöhe $\mathrm{W}_{\text {: }}$ unterscheiden. Den absoluten Betrag der Differenz dieser beiden Werte werden wir den Regressionsfehler nennen $S_{i}=\left|W_{i}-V_{i}\right|$. Wir möchten nun den Quotienten X so bestimmen, dass die Summe der Quadrate der Regressionsfehler für alle Hunde möglichst klein ist, d.h.

$$
\Sigma \mathrm{S}_{\mathrm{i}}^{2}=\Sigma\left(\mathrm{W}_{\mathrm{i}}-\mathrm{V}_{\mathrm{i}}\right)^{2}=\Sigma\left(\mathrm{W}_{\mathrm{i}}-\mathrm{X} \cdot \mathrm{D}_{\mathrm{i}}\right)^{2}=\text { Minimum. }
$$

Diese Bedingung ist erfüllt für

$$
\mathrm{X}=\Sigma \mathrm{D}_{\mathrm{i}} \cdot \mathrm{W}_{\mathrm{i}} / \Sigma \mathrm{D}_{\mathrm{i}}^{2}
$$

Nach einer kleinen Rechnung erhält man den Wert $\mathrm{X}=0,67$. Wie aus obiger Formel ersichtlich ist, ist der vom statistischen Standpunkt aus beste Quotient X demnach nicht als Verhältnis der Mittelwerte, oder an-

1) Widerrist nennt man den zwischen den Schulterblattknorpeln höchstgelegenen Rückenteil, für welchen die Dornfortsätze der ersten Brustwirbel das Knochenskelett darstellen. 
Tabelle 1.

Die reellen und nach Formeln (1) - (5) berechneten Widerristhöhen.

\begin{tabular}{|c|c|c|c|c|c|c|c|c|c|}
\hline \multirow{3}{*}{ in. } & \multirow{3}{*}{$\begin{array}{l}\text { Rasse, Inventar-Mr. } \\
\text { und Geschlecht }\end{array}$} & \multirow{3}{*}{$\begin{array}{l}\text { Innere } \\
\text { Hirnhöh- } \\
\text { lenlänge } \\
\text { /D/ In mm }\end{array}$} & \multicolumn{6}{|c|}{ Widerristhöhe $/$ il/ in om } & \multirow{3}{*}{$\begin{array}{c}\text { Verhält- } \\
\text { nis } \\
\text { W/D }\end{array}$} \\
\hline & & & \multirow{2}{*}{ reelle } & \multicolumn{5}{|c|}{ berechnet nach Formel } & \\
\hline & & & & $/ 1 /$ & 121 & $/ 3 /$ & 141 & 151 & \\
\hline 1 & Ratle Pinscher 56 - $q$ & 52,0 & 20,0 & 34,8 & 21,6 & 26,7 & 17,2 & 27,2 & 0,385 \\
\hline 2 & Pekinese e6 - 6 & 55,0 & 26,0 & 36,9 & 24,7 & 28,6 & 21,1 & 28,8 & 0,473 \\
\hline 3 & Grossspltz $47-q$ & 68,0 & 38,0 & 45,6 & 37,9 & 38,2 & 37,1 & 37,4 & 0,559 \\
\hline 4 & Grossspitz $55-6$ & 66,0 & 38,5 & 44,2 & 35,9 & 36,6 & 34,7 & 35,9 & 0,583 \\
\hline 5 & Poln. Tlefland-Schäferhund $1 / 74-6$ & 79,0 & 48,0 & 52,9 & 49,1 & 47,9 & 49,5 & 46,6 & 0,608 \\
\hline 6 & Poln. THefland-Sohäferhund $85-q$ & 78,5 & 52,0 & 52,6 & 48,6 & 47,5 & 49,0 & 45,1 & 0,662 \\
\hline 7 & Soottish Terrier $87-6$ & 74,0 & 31,0 & 49,6 & 44,0 & 43,4 & 44,0 & 42,2 & 0,419 \\
\hline 8 & Skye-Terrier $63-q$ & 70,0 & 31,0 & 46,9 & 39,9 & 39,9 & 39,4 & 38,9 & 0,443 \\
\hline 9 & Poxterrier $89-q$ & 73,0 & 45,0 & 48,9 & 43,0 & 42,5 & 42,8 & 41,3 & 0,616 \\
\hline 10 & Airedale-Terrier $5-q$ & 91,0 & 55,0 & 61,0 & 61,3 & 60,2 & 62,0 & 59,2 & 0,604 \\
\hline 11 & Deutsch Urahthaar $7-6$ & 86,0 & 56,0 & 57,6 & 56,2 & 54,9 & 56,9 & 53,6 & 0,651 \\
\hline 12 & Deursch Kurshaar $73-8$ & 87,0 & 56,0 & 58,3 & 57,2 & 55,9 & 58,0 & 54,7 & 0,644 \\
\hline 13 & Spanischer 3paniel $54-6$ & 92,0 & 57,5 & 51,6 & 62,3 & 61,3 & 63,0 & 60,4 & 0,625 \\
\hline 14 & Ir1sh Setter $53-6$ & 86,0 & 58,0 & 57,6 & 56,2 & 54,9 & 56,9 & 53,6 & 0,674 \\
\hline 15 & Inish setter $80-8$ & 93,0 & 63,5 & 62,3 & 63,3 & 62,4 & 64,0 & 61,7 & 0,683 \\
\hline 16 & Boxer $84-6$ & 78,0 & 56,0 & 52,3 & 48,0 & 47,0 & 48,4 & 45,7 & 0,718 \\
\hline 17 & Boxer $81-\delta$ & 87,0 & 60,0 & 58,3 & 57,2 & $55,9 \cdot$ & 58,0 & 54,7 & 0,690 \\
\hline 18 & Boxer $29-\delta$ & 88,0 & 51,0 & 59,0 & 58,2 & 57,0 & 59,0 & 55,8 & 0,693 \\
\hline 19 & R1esenschnauzer $93-q$ & 99,0 & 70,0 & 60,3 & 69,4 & 69,3 & 69,7 & 69,5 & 0,707 \\
\hline 20 & Poln. Bergland-Schäferuund $2 / 92-\delta$ & 99,0 & 70,0 & 66,3 & 69,4 & 69,3 & 69,7 & 69,5 & 0,707 \\
\hline 21 & Berahardiner $76-q$ & 92,0 & 67,0 & 61,6 & 62,3 & 61,3 & 63,0 & 60,4 & 0,728 \\
\hline 22 & Bernhardiner $71-\delta$ & 96,0 & 68,0 & 64,3 & 66,3 & 65,8 & 66,9 & 65,5 & 0,708 \\
\hline 23 & Bernhardiner $72-\delta$ & 106,0 & 69,5 & 71,0 & 76,5 & 77,9 & 76,0 & 80,0 & 0,656 \\
\hline 24 & Bernhardiner $90-\delta$ & 96,0 & 73,0 & 64,3 & 66,3 & 55,8 & 66,9 & 65,5 & 0,760 \\
\hline 25 & Bernhardiner $13-6$ & 100,0 & 75,0 & 67,0 & 70,4 & 70,5 & 70,6 & 70,9 & 0,750 \\
\hline 26 & Bernhardiner $32-\delta$ & 115,0 & 76,0 & 77,1 & 85,6 & 89,9 & 83,5 & 95,8 & 0,661 \\
\hline 27 & Bernherdiner $33-8$ & 104,0 & 80,0 & 69,7 & 74,5 & 75,4 & 74,2 & 76,8 & 0,769 \\
\hline 28 & Deutsche Dogge $21-\delta$ & 105,0 & 75,0 & 70,4 & 75,5 & 76,7 & 75,1 & 78,4 & 0,714 \\
\hline 29 & Deutscher Schäferhund $6-\delta$ & 93,0 & 58,0 & 62,3 & 63,3 & 62,4 & 64,0 & 61,7 & $c, 524$ \\
\hline 30 & Deutscher Sohäferhund $16-\delta$ & 95,0 & 64,0 & 63,7 & 65,3 & 64,7 & 65,9 & 64,2 & 0,674 \\
\hline 31 & Deutscher schäferhund $83-\delta$ & 96,0 & $68^{\prime}, 0$ & 54,3 & 66,3 & 65,8 & 66,9 & 65,5 & 0,708 \\
\hline 32 & Collie $67-q$ & 90,0 & 62,0 & 60,3 & 60,2 & 59,1 & 61,0 & 58,1 & 0,689 \\
\hline 33 & Collie $91-\delta$ & 94,0 & 67,0 & 63,0 & 64,3 & 63,5 & 64,9 & 62,9 & 0,713 \\
\hline 34 & Collie $61-\delta$ & 96,0 & 71,0 & 64,3 & 66,3 & 65,8 & 66,9 & 65,5 & 0,740 \\
\hline 35 & Coll1e $88-\sigma$ & 101,0 & 71,0 & 67,7 & 71,4 & 71,7 & 71,5 & 72,4 & 0,703 \\
\hline & Durchschn1ttsfehler & & & 5,2 & 3,5 & 4,0 & 3,5 & 4,6 & \\
\hline & Standardfehler & & & 6,8 & 4,6 & 5,2 & 4,5 & 6,0 & \\
\hline & Nult1pler Korrelationskoeffizient & & & 89,35 & $95,1 \%$ & $93,7 \%$ & 95,35 & 91,75 & \\
\hline
\end{tabular}


ders gesagt, nicht als Verhältnis der Merkmalssummen $\mathrm{zu}$ berechnen. Die grosse Übereinstimmung beider Zahlenwerte in unserem Fall ist rein zufällig.

Der Quotient X $=0,67$ erlaubt es mittels der inneren Hirnhöhlenlänge $\mathrm{D}$ die Widerristhöhe $\mathrm{W}$ eines Hundes zu berechnen, und zwar mit einem Durchschnittsfehler von $5,2 \mathrm{~cm}$ und einem Standardfehler von 6,8 $\mathrm{cm}$. Dies ist nicht die beste Approximation der Widerristhöhe, wie aus



Abb. 1. Darstellung der Abhängigkeit zwischen innerer Hirnhöhlenlänge (D) und Widerristhöhe (W) des Hundes.

Abb. 1 ersichtlich. Auf Abb. 1 sind die Punkte, die die wahren Messungen darstellen ${ }^{2}$ ), angegeben; mit einer Strich-Punkt-Linie ist die Gerade

(1) $\quad \mathrm{W}=0,67 \cdot \mathrm{D}$

dargestellt. Der Korrelationskoeffizient dieser Regressionsgeraden keträgt $89,3 \%$. Allgemeinverständlich gesagt würde dies bedeuten, diss etwa $89,3 \%$ der Variabilität des W durch Gleichung (1) „aufgenommen" oder ,erklärt” wird.

Wie aus Abb. 1 ersichtlich, kann die Approximation verbessert werden, wenn die Regressionsgerade im zur Uhrzeigerrichtung entgegencesetzten Sinne gedreht wird. Mathematisch bedeutet das, dass man die Bedingung, die Regressionsgerade soll durch den Nullpunkt des Koorlinatensystems gehen, fallen lässt. Wir werden jetzt solche Parameter X

2) Die Zahl der Punkte auf Abb. 1 beträgt nur 33, da zwei Punktpaare (Hurde Nr. 19 und 20, sowie Nr. 22 und 31) zusammenfallen. 
und $\mathrm{Y}$ suchen, damit $\mathrm{W}_{\mathrm{i}}=\mathrm{X} \cdot \mathrm{D}_{\mathrm{i}}+\mathrm{Y}$ wird. Wie vorher fordern wir $\Sigma \mathrm{S}_{\mathrm{i}}^{2}=\Sigma\left(\mathrm{W}_{\mathrm{i}}-\mathrm{V}_{\mathrm{i}}\right)^{2}=\Sigma\left(\mathrm{W}_{\mathrm{i}}-\mathrm{X} \cdot \mathrm{D}_{\mathrm{i}}-\mathrm{Y}\right)^{2}=$ Minimum.

Diese Bedingung ist erfüllt wenn $\mathrm{X}$ und $\mathrm{Y}$ nach den folgenden Formeln berechnet werden:

$$
\begin{aligned}
\mathrm{X} & =\frac{\mathrm{N} \Sigma \mathrm{D}_{\mathrm{i}} \cdot \mathrm{W}_{\mathrm{i}}-\Sigma \mathrm{D}_{\mathrm{i}} \cdot \Sigma \mathrm{W}_{\mathrm{i}}}{\mathrm{N} \Sigma \mathrm{D}_{\mathrm{i}}^{2}-\left(\sum \mathrm{D}_{\mathrm{i}}\right)^{2}} \\
\mathrm{Y} & =\frac{1}{\mathrm{~N}}\left(\Sigma \mathrm{W}_{\mathrm{i}}-\mathrm{X} \cdot \Sigma \mathrm{D}_{\mathrm{i}}\right), \\
\mathrm{N} & =\text { Anzahl der Messungen. }
\end{aligned}
$$

In unserem Fall erhalten wir $\mathrm{X}=1,016$ und $\mathrm{Y}=-31,2$. Die gesuchte Regressionsgerade hat demzufolge die Form:

(2) $\quad \mathrm{W}=1,016 \cdot \mathrm{D}-31,2$.

Die nach Formel (2) berechnete Widerristhöhe hat einen Durchschnittsfehler von $3,5 \mathrm{~cm}$ und einen Standardfehler von $4,6 \mathrm{~cm}$. Das bedeutet, dass die Approximation besser geworden ist. Auf Abb. 1 ist die Gerade (2) durch eine gestrichelte Linie dargestellt. Der entsprechende Korrelationskoeffizient hat sich auf $95,1 \%$ erhöht. Es sind also nur noch etwa $5 \%$ der Widerristhöhenstreuung in Gleichung (2) nicht berücksichtigt.

Wir versuchten den multiplen Korrelationskoeffizienten zu vergrössern, indem wir noch die folgenden Regressionsformeln ausprobierten:

$$
\mathrm{W}=\mathrm{X} \cdot \mathrm{D}^{2}+\mathrm{Y}, \quad \mathrm{W}=\mathrm{X} \cdot \mathrm{D}^{2}+\mathrm{Y} \cdot \mathrm{D}+\mathrm{Z}, \quad \mathrm{W}=\mathrm{X} \cdot \exp (\mathrm{Y} \cdot \mathrm{D}),
$$

$\mathrm{e}=$ Basis der natürlichen Logarithmen.

Die Berechnungen haben die folgenden Ergebnisse ergeben:

(3) $\mathrm{W}=0,006 \cdot \mathrm{D}^{2}+10,5$

(4) $\quad \mathrm{W}=-0,004 \cdot \mathrm{D}^{2}+1,72 \cdot \mathrm{D}-61,4$

(5) $\quad \mathrm{W}=9,6 \cdot \mathrm{e}^{0,02} \cdot \mathrm{D} \quad(\mathrm{e}=2,71828 \ldots)$.

Alle diese Gleichungen approximieren die Abhängigkeit zwischen Widerristhöhe W und innerer Hirnhöhlenlänge D besser als der Quotient X (Gleichung (1)). Jedoch nur Formel (4) gibt bessere Ergebnisse als Formel (2). Den Verlauf von (4) kann man auf Abb. 1 verfolgen (stetige Linie).

In Tabelle 1 sind die Werte der reellen Widerristhöhen der Hunde neben den aus den Formeln (1)-(5) errechneten zusammengestellt. Aus dieser Tabelle und aus Abb. 1 kann man den folgenden praktischen Hinweis ziehen. Zwar ist nach Gleichung (4) die Widerristhöhe am besten approximiert, aber der Unterschied zwischen den Ergebnissen der Formeln (4) und (2) ist nicht gross und der Rechenaufwand mit Formel (2) viel geringer, also folgt daraus, dass es praktisch angebracht sei, nach Formel (2) zu rechnen.

Ein Rechenbeispiel sei noch angegeben. Für Hund Nr. 3 (Grossspitz) ist die innere Hirnhöhlenlänge $\mathrm{D}=68 \mathrm{~mm}$. Nach Formel (2) erhalten wir demnach die Widerristhöhe von $\left.\mathrm{W}=1,016 \cdot 68-31,2=37,88 \mathrm{~cm} .{ }^{3}\right)$ Die gemessene, reelle Widerristhöhe dieses Hundes beträgt $38,0 \mathrm{~cm}$.

\footnotetext{
$\left.{ }^{3}\right)$ In Tabelle 1 sind die errechneten Werte auf eine Dezimalstelle abgerundet.
} 


\section{SCHRIFTTUM}

1. Boessneck J., 1956: Ein Beitrag zur Errechnung der Widerristhöhe nach Metapodienmassen bei Rindern. Ztschr. Tierzücht. Züchtungsbiol., 68, 1: 75-90.

2. C a 1 k in V. I., 1961: Izmenčivost' metapodii u oviec. Biul. Mosk. Obšč. Isp. Prirody Otd. Biol., 66, 5: 115-132.

3. El andt R., 1964: Statystyka matematyczna w zastosowaniu do doświadczalnicstwa rolniczego. Państw. Wyd. Nauk. Wroclaw.

4. K i e s ew a 1 t er L., 1888: Skelettmessungen am Pferde. Dissert. Leipzig.

5. Koudelka F., 1885: Das Verhältniss der Ossa longa zur Skeletthöhe bei den Säugetieren. Verhandl. d. naturforsch. Ver. in Brünn, 24, 1: 127-131.

6. M üller H. H., 1955: Bestimmung der Höhe im Widerrist bei Pferden. Jhrschr. Mitteldeutsche Vorgesch., 39: 240-244.

7. Perkal J., 1963: Matematyka dla przyrodników i rolników. Państw. Wyd. Nauk., 2: 1-314. Warszawa.

8. Wit t̂ W. O., 1952: Lošadi Pazyrykskih kurganov. Soviets. Archeol., 16.

9. Wyrost P. \& Kucharczyk J., 1964: Analiza przydatności niektórych parametrów czaszki psa do jej taksonomicznej oceny. Jubil. Zjazd Morfologów Polskich, Gdańsk 20-23.IX.64. Streszczenie referatów, 150.

Eingang des Ms., 27 November 1965.

Landwirtschaftliche Hochschule, Lehrstuhl für Anatomie der Tiere und

Wrocław Universität,

Wroclaw 12, Kożuchowska $1-3$. Lehrstuhl für Statistik, Wrocław, Unniwersytecka 22

Piotr WYROST i Jerzy KUCHARCZYK

\section{PRÓBA OKRESLENIA WYSOKOSCI PSA W KŁĘBIE NA PODSTAWIE WEWNĘTRZNEJ DEUGOSCI JEGO PUSZKI MOZGOWEJ}

\section{Streszczenie}

Badania nad określeniem wysokości psa w kłębie na podstawie wewnętrznej długości jego puszki mózgowej przeprowadzono na 35 dorosłych psach 19 różnych ras obu płci (9 samic i 26 samców) (Tab. 1).

W badaniach tych, po uwzględnieniu wysokiej korelacji $(95,1 \%)$ miẹdzy wewnętrzną długością puszki mózgowej (Basion - Ethmoideum), a wysokością psa w kłębie, zastosowano metody statystycznej regresji. Z pięciu ułożonych równań [(1), (2), (3), (4) i (5)], autorzy za najbardziej przydatne do stosowania w praktyce uważają równanie (2):

$$
\mathrm{W}=1,016 \cdot \mathrm{D}-31,2
$$

w którym W oznacza wartość poszukiwanej wysokości psa w kłębie w cm, a D znaną wewnętrzną długość puszki mózgowej wyrażoną w $\mathrm{mm}$.

Wzrost obliczony według tego równania odchyla się przeciętnie o $3,5 \mathrm{~cm}$ od wzrostu rzeczywistego, błą standardowy wynosi $4,6 \mathrm{~cm}$, a współczynnik korelacji wielorakiej - 95,1\%. Oznacza to, że za pomccą tego równania zmienność wzrostu rzeczywistego można wytłumaczyć w około $95 \%$. 